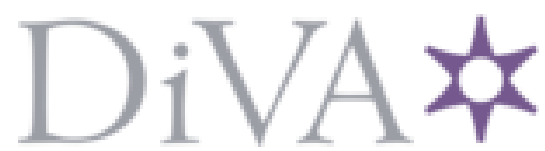

http://www.diva-portal.org

\title{
Postprint
}

This is the accepted version of a paper presented at The Applied Computational Electromagnetics Society (ACES).

Citation for the original published paper:

Ghasemifard, F., Norgren, M. (2017)

Sensor Selection via Convex Optimization in Remote Contact-free Measurement of Currents. In:

N.B. When citing this work, cite the original published paper.

Permanent link to this version:

http://urn.kb.se/resolve?urn=urn:nbn:se:kth:diva-199687 


\section{Sensor Selection via Convex Optimization in Remote Contact-free Measurement of Currents}

\author{
Fatemeh Ghasemifard \\ School of Electrical Engineering \\ KTH Royal Institute of Technology \\ Stockholm, Sweden \\ Email: fatemehg@kth.se
}

\author{
Martin Norgren \\ School of Electrical Engineering \\ KTH Royal Institute of Technology \\ Stockholm, Sweden \\ Email: mnorgren@kth.se
}

\begin{abstract}
The sensor positions have substantial effect on the accuracy of remote contact-free reconstruction of currents in power transmission lines from magnetic field data, measured by a set of sensors located under the conductors. By maximizing a performance metric, based on the Fisher information matrix, $N_{\text {opt }}$ sensors are selected from $N$ candidate sensors. The main optimization problem is changed to a convex optimization problem using a relaxation of the integer constraints. The result for optimized sensor positions for a remote current reconstruction in power transmission lines using a planar sensor array is demonstrated.
\end{abstract}

\section{INTRODUCTION}

Remote contact-free measurement of currents [1] and voltages [2] in power transmission lines could improve the reliability of the power system by providing more information about the transported power and the state of the grid [3], [4]. These techniques provide the possibility of measuring high-frequency voltages and currents, as well as, measuring voltages and currents at locations where measurement transformers cannot be installed. Hence, the contact-free technique could be a complement to the established technique of using measurement transformers.

Contact-free measurement of currents is a magnetic inverse source problems, where the currents are reconstructed using magnetic field data collected by a set of sensors located in the vicinity of the conductors [3], [5]. One significant difficulty is the influence of the magnetic field generated by other sources. In our recent work [3], the external magnetic field is modeled by an expansion into spherical harmonics, whose coefficients are determined together with the sought currents. This method can handle effectively the effect of disturbance fields, but in [3] we did not consider optimizing the sensor positions. Since the sensor positions have substantial effect on the accuracy of the current reconstruction, it is possible to reduce the effect of disturbance fields even further by finding the optimal positions of the sensors [5], [6].

In this paper, we extend our recent work [3] by sensor selection based on Convex Optimization [7]. Following the method presented in [7], we select $N_{\text {opt }}$ sensors among $N$ candidates by minimizing the negative of the determinant of the Fisher Information matrix [8]. By relaxing the integer constraints, we obtain a convex optimization problem, as explained in the next section.

\section{Sensor Selection Optimization Problem}

In the current reconstruction method proposed in [3], the $x-, y$-, and $z$-components of the magnetic field, measured by each sensor, are represented by linear combinations of the currents $\left\{I_{i}\right\}_{i=1}^{3}$ to be reconstructed and the unknown expansion coefficients, $\mathbf{A}_{\ell m}$, of the spherical harmonics. As a result, arranging all the parameters to be estimated in the vector $\mathbf{p}=\left\{\operatorname{Re}\left(I_{i}\right), \operatorname{Im}\left(I_{i}\right), \operatorname{Re}\left(\mathbf{A}_{\ell m}\right), \operatorname{Im}\left(\mathbf{A}_{\ell m}\right)\right\}_{i=1}^{3}$, where the number of elements in $\mathbf{A}_{\ell m}$ depends on $\ell_{\max }$, the truncation number in spherical harmonic expansion [3], we have

$$
\begin{aligned}
B_{j, x}=\mathbf{a}_{j, x}^{\mathrm{T}} \mathbf{p}+n_{j, x}, & j=1, \ldots, N, \\
B_{j, y}=\mathbf{a}_{j, y}^{\mathrm{T}} \mathbf{p}+n_{j, y}, & j=1, \ldots, N, \\
B_{j, z}=\mathbf{a}_{j, z}^{\mathrm{T}} \mathbf{p}+n_{j, z}, & j=1, \ldots, N,
\end{aligned}
$$

where $N$ is the number of measurement points, $\mathbf{B}_{j}=$ $\left(B_{j, x}, B_{j, y}, B_{j, z}\right)$ is the magnetic field data collected by the $j$-th sensor and $n_{j, x}, n_{j, y}, n_{j, z}$ are independent identically distributed Gaussian noise with mean zero and variance $\sigma^{2}$.

Note, if only the currents $\left\{I_{i}\right\}_{i=1}^{3}$ were to be reconstructed, we would have a biased reconstruction problem, with an unknown bias due to the disturbance field. Nevertheless, since both $\left\{I_{i}\right\}_{i=1}^{3}$ and $\mathbf{A}_{\ell m}$ are reconstructed, the reconstruction problem is unbiased. Thus, the Cramér-Rao lower bound (CRLB) theorem $\mathbf{C}_{\mathbf{p}} \succeq \mathbf{I}^{-1}$, where $\mathbf{C}_{\mathbf{p}}$ is the covariance matrix of the estimated parameters and $\mathbf{I}$ is the Fisher information matrix, is applicable. As a result, the Fisher information matrix could be a metric for the performance of the parameter estimation. In fact, the efficiency of the current reconstruction method is expected to improve by maximizing $\mathbf{I}$ in some sense. However, in general, it is not possible to find an optimal I. Instead, a D-optimal solution [7] is sought, which means maximizing $\log \operatorname{det}(\mathbf{I})$ or minimizing $-\log \operatorname{det}(\mathbf{I})$.

The Fisher Information matrix, I, is defined as [8], [9]

$$
\mathbf{I}=\sum_{j=1}^{N} \mathbf{I}_{j},
$$

where $\mathbf{I}_{j}$, the Fisher information matrix corresponding to the $j$-th sensor, is defined as

$$
\mathbf{I}_{j}=\frac{2}{\sigma^{2}} \operatorname{Re}\left\{\left(\nabla_{\mathbf{p}} \mathbf{B}_{j}\right)^{\mathrm{H}} \nabla_{\mathbf{p}} \mathbf{B}_{j}\right\} .
$$


In (5), $\mathrm{H}$ denotes the conjugate transpose and $\nabla_{\mathbf{p}} \mathbf{B}_{j}=$ $\left[\begin{array}{lll}\mathbf{a}_{j, x} & \mathbf{a}_{j, y} & \mathbf{a}_{j, z}\end{array}\right]^{\mathrm{T}}$. Now, the sensor selection problem of finding $N_{\text {opt }}$ D-optimal sensors from $N$ candidates can be formulated as

$$
\begin{array}{ll}
\underset{w_{j}}{\operatorname{minimize}} & -\log \operatorname{det}\left(\frac{\sum_{j=1}^{N} w_{j} \mathbf{I}_{j}}{N_{\mathrm{opt}}}\right) \\
\text { subject to } & w_{j} \in\{0,1\}, j=1, \ldots, N, \\
& \sum_{j=1}^{N} w_{j}=N_{\mathrm{opt}} .
\end{array}
$$

In (6), $w_{j}=1$ means the $j$-th sensor should be used and $w_{j}=0$ means the $j$-th sensor should be removed. There are $\left(\begin{array}{c}N \\ N_{\text {opt }}\end{array}\right)$ combinations for selecting $N_{\text {opt }}$ out of $N$ sensors. Thus, (6) can be solved only for small $N$ by an exhaustive search. However, when $N$ is large, a relaxation of the integer constraints [7] turns (6) into a convex optimization problem, which can be readily solved using the CVX package [10]. In the relaxation, we assume that all $N$ sensors are used to measure the magnetic field and that the weight on the $j$ th sensor is $\lambda_{j}=w_{j} / N_{\text {opt }}$. Thus, the relaxed problem is formulated as

$$
\begin{array}{ll}
\underset{w_{j}}{\operatorname{minimize}} & -\log \operatorname{det}\left(\sum_{j=1}^{N} \lambda_{j} \mathbf{I}_{j}\right) \\
\text { subject to } & 0 \leq \lambda_{j} \leq 1 / N_{\text {opt }}, j=1, \ldots, N, \\
& \sum_{j=1}^{N} \lambda_{j}=1 .
\end{array}
$$

The upper bound on $\lambda_{j}$ ensures that at least $N_{\text {opt }}$ weights are non-zero.

\section{RESULT}

Consider the example of reconstructing currents in three power transmission lines with a magnetic dipole and three line sources as external sources presented in [3]. The potential sensor positions are $21 \times 21$ measurement points (blue crosses in Fig. 1) distributed equidistantly with the separation $0.2 \mathrm{~m}$ in both $x$ - and $y$-direction over a square measurement plane located at the height $z=1.5 \mathrm{~m}$ and with its boundaries at $x, y= \pm 2 \mathrm{~m}$. CVX package [10] is used to solve the relaxed convex optimization problem (7) with $N=441$ and $N_{\text {opt }}=216$. It should be mentioned that calculating $\mathbf{I}_{j}$ was done by setting $\ell_{\max }=4$ for truncating the spherical harmonic expansion [3]. The result of sensor selection which is shown in Fig. 1 by red circles were 224 sensor positions with $\lambda_{j}>10^{-3}$. It means the sensors with $\lambda_{j}<10^{-3}$ can be removed. The currents were estimated using 224 sensors located at optimal sensor positions rather than using 441 sensors, but the variance of the estimated currents remained almost the same as the case when 441 sensors was used. However, the speed of the current estimation improved a lot.

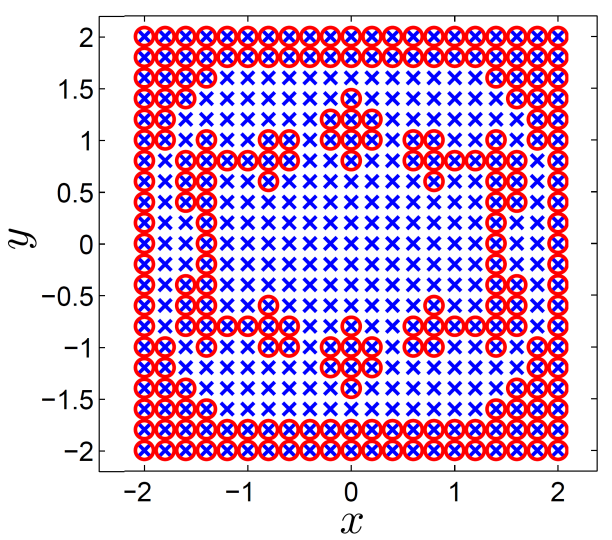

Fig. 1: The potential positions of the sensors (blue crosses) and the optimal positions of the sensors (red circles).

\section{CONCLUSIONS AND FUTURE WORK}

In this paper, a method for sensor selection based on convex optimization in remote contact-free reconstruction of currents in power transmission lines [3] was presented. The aim was to select $N_{\text {opt }}$ sensors among $N$ sensors with predetermined positions to maximize the performance of current estimation. For this purpose, the Fisher information matrix was used as a performance metric. This optimization problem could be solved only when the number of the potential sensors is small. To be able to find the solution for the large numbers of the potential sensors, the main optimization problem was altered into a convex problem by relaxing the integer constraint.

In future work, this method will be compared with other sensor selection methods, like the one in [6], and also tested with measurement data.

\section{REFERENCES}

[1] Kruphalan Tamil Selvaa, et al., "Non-contact Current Measurement in Power Transmission Lines," Procedia Technology: Smart Grid Technologies, vol. 21, pp. 498-506, 2015.

[2] J. Törnqvist, Non-contact High Voltage Measurements: Modeling and Onsite Evaluation, Umeå University, Umeå, Sweden, Tech. Rep., 2012.

[3] F. Ghasemifard, M. Johansson, and M. Norgren, "Current reconstruction from magnetic field using spherical harmonic expansion to reduce impact of disturbance fields," Submitted to Inverse Problems in Sci. and Eng., pp. 1-15, 2016.

[4] F. Ghasemifard and M. Norgren, "Contact-free measurement of currents in two-dimensional parallel conductors using the Green identity approach," in 2016 URSI International Symposium on Electromagnetic Theory (EMTS), Aug. 2016, pp. 338-340.

[5] M. Norgren, "Explicit reconstruction of line-currents and their positions in a two-dimentional parallel conductor structure," Inverse Problems in Sci. and Eng., vol. 22, no. 7, pp. 1196-1213, 2013.

[6] Z. Zhang Z, L. Di Rienzo, "Optimization of magnetic sensor arrays for current measurement based on swarm intelligence and D-optimality," COMPEL - Int. J. Comput. Math. Electr. Electron. Eng., vol. 28, pp. 1179-1190, 2009.

[7] S. Joshi and S. Boyd, "Sensor Selection via Convex Optimization," IEEE Trans. Signal Process., vol. 57, no. 2, pp. 451-462, 2009.

[8] Kay SM. Fundamentals of Statistical Signal Processing, Estimation Theory. NJ: Prentice-Hall; 1993.

[9] O. Talcoth and T. Rylander, "Sensor selection in magnetic tracking based on convex optimization," Electronics Lett., vol. 49, no. 1, pp. 15-16, 2013.

[10] Grant, M., and Boyd, S.: "CVX: Matlab Software for Disciplined Convex Programming," version 2.1, October 2016, http://cvxr.com/cvx. 